\section{Chile: protección social de la salud}

\author{
Rafael Urriola ${ }^{1}$
}

Palabras clave: cobertura universal, costos de la atención de salud, extensión de la cobertura, financiación de la salud, seguros de salud, Chile.

1 FONASA. La correspondencia debe ser dirigida a Rafael Urriola, FONASA, Monjitas 665, Santiago, Chile. Dirección electrónica: rurriola@ fonasa.cl; teléfono: 5626614807 .
Este artículo tiene por objeto contribuir a una reflexión acerca de la protección social de la salud en Chile. Se ofrece una revisión conceptual inicial para evitar interpretaciones que puedan desembocar en otros conceptos relacionados pero no idénticos, tales como equidad, desigualdad o cohesión social. Luego se examina la protección social a la luz de la experiencia chilena, especialmente desde la década de los noventa, en tres ámbitos principales.

El primero se relaciona con la cobertura de la seguridad social en salud que goza la población del territorio nacional y se exponen los aspectos legales, estadísticas y requisitos de la legislación actual para lograr o dificultar la universalidad. El segundo ámbito identifica los problemas sanitarios en los cuales la población asegurada puede sentir que dispone de derechos de acceso con garantías explícitas o razonables, es decir, lo que hemos llamado cobertura vertical. En tercer lugar, el documento aborda la protección financiera, otro componente principal de la protección social. En este campo se examinan aspectos relacionados con las fuentes de financiamiento, como el aporte fiscal, las cuotas de la seguridad social y el impacto de los gastos de bolsillo en los hogares.

Finalmente, se revisa la legislación reciente que ha ampliado la cobertura financiera y se describen los programas especiales instaurados por el seguro público chileno desde la década de los noventa para mejorar este tipo de cobertura. La última sección se centra en las conclusiones.

\section{PROTECCIÓN SOCIAL: EL MARCO CONCEPTUAL}

Los derechos sociales -entre los que se encuentra el derecho a la salud o a la protección de la salud- llamados "de segunda generación" corresponden a demandas formuladas para corregir las inequidades y carencias que se hicieron manifiestas con el surgimiento de los sistemas de gobierno republicanos. Lo anterior se combinó con la "cuestión social" que emergió después de la Revolución Industrial y que se institucionalizó con mayor fuerza en Europa luego del triunfo del bolchevismo en Rusia.

Un gran debate vinculado a los derechos económicos, sociales y culturales es el de la 'justiciabilidad' o la posibilidad de que un juez dictamine que debe haber una reparación por la violación de un 
derecho económico o social, como ocurre con la violación de los derechos civiles y políticos, conocidos como "de primera generación". Es interesante tener presente que en muchos países se toman cada vez más decisiones judiciales que obligan a los Estados a proteger determinados derechos económicos y sociales como los que se refieren a no disminuir las pensiones o a la obligación de alimentar a las familias (1).

La Constitución de Chile de 1980 mantuvo formalmente los derechos sociales señalando que en salud existían dos sistemas: uno privado y otro público, que debía esencialmente cubrir a quienes no tenían recursos.

Según F. Zúñiga (2), esta Constitución respalda el carácter subsidiario del Estado, es decir, que concibe esencialmente la actividad estatal en los ámbitos en donde no hay oferta privada. En esta manera de interpretar la Constitución, los derechos sociales son optativos y dependen, más bien, de las definiciones ideológicas o políticas de cada gobierno, antes que de un mandato constitucional explícito. Zúñiga considera necesario, en cambio, un Estado social y democrático de derecho que incluya "derechos de participación (derechos políticos) y de prestación (derechos económicos, sociales y culturales) [...] en que cada uno, en cuanto miembro de la comunidad política, obtiene del Estado un conjunto de bienes y servicios comunitarios mínimos." En efecto, el plan de Acceso Universal de Garantías Explícitas de salud (AUGE) de Chile - que se comentará en este documento- tiene como característica fundamental la obligatoriedad del asegurador de cumplir con el plan. De no hacerlo, arriesga sanciones legales y pecuniarias.

Por otra parte, la crisis económica y social, que no excluyó a Chile y que afectó en los años ochenta a América Latina, provocó una sensación de crisis relacionada con las propias intervenciones de los organismos internacionales ya que, socialmente, la mayor parte de los indicadores relevantes se deterioraron en prácticamente todos los países de la Región. Estas mismas instituciones generaron fuertes autocríticas y nuevas definiciones en cuanto a los aspectos sociales del ajuste, que empezaron por desestimar la eficacia de la llamada "política del chorreo" que no funcionó como se enunciaba en los principios básicos del llamado Consenso de Washington (3-5).

Después de ese momento, es decir desde principios de la década de los noventa, surgieron varios enfoques para renovar las políticas sociales. No está demás mencionar que este debate puede incluirse en el marco general de las políticas referentes al "estado de bienestar", que han involucrado opiniones de agencias internacionales y autoridades sectoriales, internacionales y nacionales.
Por último, es posible centrar el debate en dos enfoques principales: uno caracterizado como manejo social del riesgo (6), que tiene por objeto mitigar las repercusiones de crisis o perturbaciones covariantes (las cuales afectan a muchas personas y pueden asociarse, por ejemplo, a la crisis regional de los años ochenta que la transformaron en una "década perdida") o idiosincrásicas (que repercuten o se producen en grupos reducidos, como una muerte o una enfermedad catastrófica). Un estudio sobre esta estrategia de manejo de riesgo (7) plantea que ésta tiene tres componentes: reducción del riesgo, alivio del riesgo y enfrentamiento del riesgo. El peso del aporte privado para reducir los riesgos sociales, subyacente en este enfoque, obedece a que, durante la recesión, el gasto en los pobres fue muy procíclico, aunque en teoría debería haber sido anticíclico, a fin de protegerlos de la perturbación macroeconómica adversa. Es decir, la propuesta de intensificar los aportes privados a la seguridad social se basa en que los mecanismos de protección resultan insuficientes cuando más se necesitan debido a la inflexibilidad en la conducción macroeconómica que ha primado en la Región desde la década de los noventa (8). En definitiva, este punto de vista conduce a anteponer las políticas focalizadas a las universales $y$, en el plano del aseguramiento, la de desestimar la eficacia de los mecanismos basados en la solidaridad y hacer hincapié, en cambio, en la responsabilidad individual para asegurarse contra los riesgos.

El otro enfoque podría resumirse en que la acción del Estado o la participación obligatoria en una mancomunación de riesgos es pertinente aunque los mecanismos de mercado no existan, se colapsen o sean disfuncionales, puesto que las asimetrías de información y las fallas de mercado son inherentes a los mercados de aseguramiento y no situaciones excepcionales. En esos términos, la acción pública regulatoria, el aseguramiento público, o el aseguramiento social con aseguradores privados pero con mecanismos de financiamiento obligatorio y regulaciones que garanticen la diversificación del riesgo, permiten enfrentar la selección del riesgo e incrementar la eficiencia de estos mercados, al propiciar la estabilidad del aseguramiento. Cuando se opta por el financiamiento solidario, los objetivos, además, son redistributivos y es posible establecer subsidios cruzados entre estratos de ingreso, grupos etarios, de riesgo, $\mathrm{u}$ otros (9).

En este sentido, lo planteado por Titelman y Uthoff es relevante para potenciar la protección social, en particular, si entendemos que la salud es un derecho y no un bien "deseable" sujeto a los recursos personales o a las donaciones de quienes tengan a bien hacerlas. "En entornos económicos caracterizados por más incertidumbre y volatilidad, lo que 
se traduce en mayores riesgos sociales, las reformas de la seguridad social deben conciliar las funciones de aseguramiento y ahorro con las de redistribución y solidaridad. [. . .] En el caso del sector de la salud, los seguros sociales pasan a ser una forma eficiente de organizar el financiamiento. Para superar la actual segmentación poblacional entre diferentes subsistemas de protección social en materia de salud, es necesario independizar la pertenencia al seguro de la forma de inserción laboral. Esto es, el seguro debe cobijar a trabajadores dependientes e independientes y a la población sin capacidad de contribución" (10).

La Organización Internacional del Trabajo (OIT), por su parte sostiene que "la protección social no es facultativa sino un componente necesario de las estrategias de lucha contra la pobreza. Nuestra primerísima prioridad es llegar a aquellos ciudadanos no cubiertos por ningún sistema. Sólo esto representa un enorme reto y no se le puede hacer frente de la noche a la mañana. Sin embargo, teniendo en mente nuestros objetivos a largo plazo, podemos dirigir nuestras acciones a corto plazo hacia la universalidad y hacia sistemas integrales de protección social. Éste es el espíritu de nuestra Campaña mundial sobre seguridad social y cobertura para todos" (11).

Recientemente, la Unión Europea (UE) y la propia Comisión Económica para América Latina y el Caribe (CEPAL) incorporaron en sus agendas un concepto útil y complementario para evaluar la acción pública a favor de la protección social: la cohesión social. En Europa, la idea de cohesión social se ha institucionalizado por la necesidad de examinar en profundidad los sentimientos de pertenencia, confianza y seguridad en relación a esa comunidad. El documento que fijó la estrategia de la UE al respecto (12) exigió una redefinición posterior que concluye que cohesión social es: "la capacidad de la sociedad para asegurar de forma permanente el bienestar de todos sus miembros por medio del igual acceso a los recursos disponibles, el respeto a la dignidad dentro de la diversidad, la autonomía individual y colectiva, y la participación responsable" (13).

La cohesión social, en una visión más funcionalista -en el sentido sociológico del término-, se ha definido también como la promoción de comunidades estables, cooperativas y durables. Un ejemplo de esta visión lo constituye el Plan de Inclusión Social del Reino Unido (14) que basa su forma de acción en tres líneas principales: erradicar la pobreza en los niños, romper las barreras de acceso al trabajo de las personas en edad de trabajar y reformular las políticas hacia los barrios marginalizados o con mayores carencias. En esta última versión priman las ideas de "recuperar la armonía" antes que fortalecer las potencialidades comunitarias, que es el sentido principal de la definición ac- tual de la UE. Algunas de las interpretaciones de cohesión social hacen alusión a los sentimientos de pertenencia y a las identidades valóricas, lo que restringe (quizás hasta contradice) el alcance de la definición dada por la UE en tanto ésta reconoce y valora la diversidad. No es posible entender el debate europeo sin recordar que se trata de sociedades con fuerte presencia de migraciones históricas que alcanzan a proporciones significativas de la población y que, además, provienen de culturas muy diversas y que no se han adaptado fácilmente a los patrones culturales occidentales europeos. Quizás el concepto sea útil en América Latina y Chile al momento de reflexionar acerca de las maneras en que los pueblos originarios acceden a la salud.

Existen, además, otras definiciones que ponen el acento en las capacidades para vivir y reaccionar de manera común en función de principios societales, por ejemplo, la democracia. No obstante, esta acepción no responde a la interrogante sobre esos objetivos que podrían no ser comunes y que, en sí mismos, pueden ser excluyentes. Cabe notar que, de otro lado, el término exclusión se usa como aproximación válida a la no cohesión aunque tiene diferentes acepciones relacionadas con la forma de exclusión: económica (en relación al consumo, al trabajo o a los servicios); social (en relación a la vivienda o a la protección social en general); cultural (deserción escolar, analfabetismo en cualquiera de sus formas, abandono de la identidad básica/natal); física (alcoholismo, discapacidad física); y legal (sin acceso al sistema de justicia, indocumentado). De cualquier modo, al intervenir positivamente en las redes que determinan los procesos de exclusión se avanza en la cohesión social y, en este ámbito, la protección social es uno de los ejes para hacerlo.

$\mathrm{Al}$ examinar el conjunto de definiciones y alcances de la protección social y otros términos afines, en este documento se ha optado por entender el concepto de protección social de la salud como suficientemente explicitado a la luz de tres dimensiones e indicadores principales: la cobertura horizontal (proporción de la población que tiene cobertura de la seguridad social en salud); la cobertura vertical (a qué prestaciones tienen derecho efectivo, oportuno y razonablemente garantizado esos afiliados); y la protección financiera (en qué medida los gastos totales o complementarios en salud no exacerban la desigualdad, sea en ingresos o en estados de salud).

Cabe notar que en los estudios sobre la salud se han desarrollado también otros conceptos afines como el de determinantes sociales y equidad horizontal y vertical. Para asegurar la verdadera igualdad no basta que la sociedad ofrezca posibilidades (prestaciones de salud) de uso a las personas. Si una persona presenta el mismo estado de salud que otra 
persona, el principio de equidad horizontal exige que ambos reciban el mismo tratamiento. Sin embargo, si uno de ellos tiene menos instrucción que el otro y no asume (o no comprende) las instrucciones de su médico, entonces debe recibir tratamientos diferentes. En esta perspectiva es que se introduce la noción de determinantes sociales, la cual implica que a una persona con más necesidades deben dársele tratamientos adicionales.

Por su parte, la equidad puede entenderse tanto desde el punto de vista de la utilización de servicios como de los modos de financiarlos. En el primer caso, la equidad consiste en que el acceso a los servicios de salud tenga como única condición las necesidades de salud sin tomar en cuenta situaciones económicas, sociales, étnicas, u otras. Pero, en general, los estudios sobre equidad - véase la revisión hecha por Wagstaff y Van Doorslaer- se centran en cómo financiar las prestaciones de salud de acuerdo con las capacidades de pago. Estos autores señalan que la equidad horizontal se expresa cuando todos los miembros de la sociedad con una misma capacidad de pago contribuyen de forma idéntica al financiamiento. Habría equidad vertical si, además, los pagos se organizaran de acuerdo con la capacidad de pagar de modo que los ricos paguen más que los pobres (15).

El enfoque de equidad, entonces, se centra en los modos de financiamiento y los ingresos personales. En cambio, nuestra noción de protección social tiene un énfasis institucional aunque el financiamiento se aborda como un componente que puede restringir el acceso a los servicios de salud.

\section{LA ESTRUCTURA DE LA SEGURIDAD SOCIAL Y LA PROTECCIÓN SOCIAL}

En la concepción inicial de la seguridad social se buscaba esencialmente proteger a los trabajadores. El primer fenómeno, probablemente el detonante para todos los impactos de desprotección analizados en este documento, es que las sociedades no convergieron hacia el empleo pleno ni lograron que el trabajo formal, con relaciones de dependencia, creciera proporcionalmente en relación a la población activa. Por cierto, esta discusión pierde relevancia en los sistemas de financiamiento universal con impuestos generales. En efecto, coyunturalmente, hay variaciones más rápidas del desempleo ante fluctuaciones en el ritmo de actividad económica. Asimismo, el ajuste ante la contracción es más veloz que ante la expansión, lo que en situaciones de crisis recurrentes de corto plazo ha significado tasas de desempleo crecientes. Por ejemplo, la tasa media de desempleo para la Región de América Latina y el Caribe ha aumentado de manera
CUADRO 1. Empleados sin seguro de salud según su categoría ocupacional. Chile, 2003

\begin{tabular}{lrr}
\hline \multicolumn{1}{c}{ Categoría ocupacional } & \multicolumn{1}{c}{$n$} & \multicolumn{1}{c}{$\%$} \\
\hline Patrón o empleador & 39791 & 7,6 \\
Trabajador por cuenta propia & 205961 & 39,4 \\
Empleado u obrero & & \\
$\quad$ de instituciones públicas & 17706 & 3,4 \\
Empleado u obrero del sector privado & 226599 & 43,3 \\
Otra & 32965 & 6,2 \\
$\quad$ Total & 523022 & 100,0 \\
\hline
\end{tabular}

Fuente: Encuesta Caracterización Socioeconómica Nacional (CASEN) 2003.

continua, pasando - según datos del Banco Mundial- de 6,6\% en la década de 1970 a 7,6\% en la de 1980 y a $7,9 \%$ en la de 1990 . Asimismo, los datos de la OIT muestran la misma tendencia, al pasar de tasas medias en torno a $4 \%$ entre 1950 y 1970, a tasas medias en torno a 7\% durante los decenios de 1980 y 1990 (16).

En Chile, los asalariados o trabajadores dependientes representaban $70 \%$ de la fuerza de trabajo ocupada en 1980; 72\% en 1991 y $65 \%$ en 2005 $(17,18)$. A esto se suma el aumento del trabajo por cuenta propia, la subcontratación (outsourcing) como estrategia de las grandes empresas, el trabajo a tiempo parcial y los empleos temporales. En suma, como se observa en el cuadro 1, la precarización de los empleos y la persistencia de un sector informal atenta contra la protección social universal basada en cotizaciones, lo que obliga a una participación directa del Estado para financiar la salud de la población.

Los impactos de la precarización laboral sobre la seguridad social son inmediatos. Estudios del Fondo Nacional de Salud de Chile (FONASA) indican que, como consecuencia de la inseguridad de los empleos, sólo $25 \%$ de la población afiliada paga cuotas por el año completo. Uthoff obtuvo una conclusión similar (19) en un estudio relacionado especialmente con las cotizaciones previsionales para pensiones cuya estabilidad y crecimiento pareciera sustentarse en un inexistente mercado de trabajo estable, formal y creciente.

\section{COBERTURA HORIZONTAL O POBLACIONAL DE LA SEGURIDAD SOCIAL}

Desde 1980, en el marco de la restricción de las tareas del Estado que primaban entonces, en Chile se instauró un sistema mixto de salud en el que los trabajadores podían optar por que sus cotizaciones (7\% de los ingresos) fueran administradas por aseguradoras privadas o por el seguro público, es decir el FONASA que, además, tiene por misión cubrir a las personas carentes de recursos. En cam- 
CUADRO 2. Protección de la Seguridad Social. Chile, 2004

\begin{tabular}{|c|c|c|c|c|}
\hline Subsistema & Principales beneficios & Administración & Cobertura & $\begin{array}{c}\text { Fuente de } \\
\text { financiamiento }\end{array}$ \\
\hline Pensiones & $\begin{array}{l}\text { Pensión por vejez, } \\
\text { pensión mínima } \\
\text { garantizada por el } \\
\text { Estado y pensiones } \\
\text { asistenciales }\end{array}$ & $\begin{array}{l}\text { Privada: } \\
\text { Administradoras de } \\
\text { Fondos de Pensiones } \\
\text { (AFP) } \\
\text { Pública: Instituto } \\
\text { Nacional de Previsión } \\
\text { (INP) }\end{array}$ & $\begin{array}{l}61 \% \text { de los cotizantes } \\
\quad \text { (fuerza de trabajo) } \\
\text { Diciembre } 2003\end{array}$ & $\begin{array}{l}\text { 10\% cotización del } \\
\text { afiliado (o del Estado } \\
\text { en caso de pensiones } \\
\text { mínimas y pensiones } \\
\text { asistenciales) }\end{array}$ \\
\hline $\begin{array}{l}\text { Invalidez } \\
\text { y supervivencia }\end{array}$ & $\begin{array}{l}\text { Pensión por invalidez } \\
\text { o pensión de } \\
\text { supervivencia a } \\
\text { familiares en caso de } \\
\text { muerte }\end{array}$ & $\begin{array}{l}\text { Privada: AFP } \\
\text { Pública: INP }\end{array}$ & s.d. ${ }^{a}$ & $\begin{array}{l}\text { Compra de seguro por } \\
\text { parte del afiliado }\end{array}$ \\
\hline Salud & $\begin{array}{l}\text { Cobertura de atención } \\
\text { médica y tratamientos }\end{array}$ & $\begin{array}{l}\text { Privada: ISAPRE } \\
\text { Pública: FONASA }\end{array}$ & $\begin{array}{l}86 \% \text { de los beneficiarios } \\
\text { (población total) } \\
\text { Diciembre } 2003\end{array}$ & $\begin{array}{l}\text { 7\% cotización del afiliado } \\
\text { (o del Estado en caso } \\
\text { de personas carentes } \\
\text { de recursos) }\end{array}$ \\
\hline Accidentes de trabajo & $\begin{array}{l}\text { Pensión por invalidez y } \\
\text { supervivencia }\end{array}$ & Mutuales de seguridad & $\begin{array}{l}7 \% \text { de los trabajadores } \\
\text { (cotizantes } \\
\text { empleados) } \\
\text { Diciembre } 2003\end{array}$ & $\begin{array}{l}\text { Compra de seguro por } \\
\text { parte del empleador }\end{array}$ \\
\hline Desempleo & $\begin{array}{l}\text { Transferencia mensual } \\
\text { con tope en caso de } \\
\text { desempleo }\end{array}$ & $\begin{array}{l}\text { Administradora de } \\
\text { Fondos de Cesantía }\end{array}$ & $\begin{array}{l}73 \% \text { de los cotizantes } \\
\text { (asalariados) } \\
\text { Junio } 2004\end{array}$ & $\begin{array}{l}\text { Cuenta individual del } \\
\text { trabajador y Fondo } \\
\text { Solidario }\end{array}$ \\
\hline
\end{tabular}

Fuente: M. Marcel (19).

${ }^{\text {a }}$ s.d. $=$ sin datos.

bio, la cobertura no es una preocupación de los sistemas privados. Por ello, en este capítulo se describe el proceso que, finalmente, ha desembocado en una creciente segmentación de los seguros.

Según M. Marcel (20), Director de Presupuestos del Ministerio de Hacienda de Chile en 2004, los fundamentos del Sistema de Protección Social se basan en el crecimiento económico sostenido, políticas sociales redistributivas y estabilidad de las instituciones democráticas. Pese a los logros relevantes en cuanto a reducción de la pobreza en el período posterior a 1990 - de alrededor de $40 \%$ a $18 \%$ de la población en 2003 (21)-, hay diversos indicadores que distan mucho de lo que puede esperarse para países que actualmente se califican como economías emergentes. Aun así, como se observa en el cuadro 2, el sistema de seguridad social de todos modos logra mayor cobertura que en países de desarrollo semejante, incluida la mayoría de los de la propia Región.

El sistema chileno de seguros de salud mixto, público y privado, en 2005 cubrió a $88 \%$ de la población distribuida de la siguiente manera: $68,6 \%$ por el seguro público, es decir, el FONASA; 16,8\% por las aseguradoras privadas, es decir, las Instituciones de Salud Previsional (ISAPRE); y 2,6\% por los seguros públicos de salud de las fuerzas armadas.
Quiénes son y por qué hay grupos poblacionales excluidos de la seguridad social es un tema central de la protección social para la Organización Panamericana de la Salud (OPS) (22). Cabe notar que en los últimos años ha cobrado especial relevancia el debate acerca del financiamiento de la seguridad social puesto que el sistema se ha verificado incapaz de incluir a un importante segmento de la población trabajadora en el sector informal. ${ }^{2}$ Tal situación ha resituado las posiciones a favor de que la salud sea esencialmente financiada con impuestos.

En Chile, para reducir los segmentos de la población excluidos de los beneficios de la seguridad social, se ha optado por modificar la legislación a favor de los grupos desprotegidos. En el caso de los trabajadores, se ha desarrollado una modalidad de cotización especial para los temporeros con un sistema de remuneración diaria. A partir de 2001, éstos han tenido derecho a extender la atención de salud en calidad de afiliados, hasta un año después de haber pagado su última cuota, con sólo acreditar

\footnotetext{
2 De alguna forma se repone el debate entre el sistema "bismarckiano" (atribuido al alemán Bismarck) que enfatiza la seguridad social para trabajadores formales y el sistema "beveridgeano" (por el inglés Beveridge) que considera que los sistemas de seguridad social deben cubrir a toda la población.
} 
60 días de cotizaciones en FONASA dentro del año. Esto significa que 200000 temporeros y sus familias (otras 200000 personas) fueron cubiertas en 2005 por FONASA. También se ha extendido a los cesantes, de los cuales 65000 han sido aceptados por el seguro público con derechos plenos (23).

De otra parte, en la calidad de beneficiarios no cotizantes de FONASA, la cobertura se extiende a las siguientes personas:

- Los familiares a cargo de los cotizantes, que cumplan con los requisitos del Decreto con Fuerza de Ley (DFL) 150. Esta condición, vigente desde la instauración del sistema mixto a principios de los años ochenta, también es válida en el seguro privado.

- La mujer embarazada, que no tiene previsión, hasta el sexto mes del nacimiento del hijo.

- Personas carentes de recursos o indigentes (FONASA A) que, por lo demás, representaron en 2005 el 34,3\% de los beneficiarios del seguro público (casi 4 millones de personas); entre ellos, a los beneficiarios de pensiones asistenciales de invalidez y ancianidad (PASIS) y a los causantes del Subsidio Único Familiar (SUF). Este subsidio es entregado por las municipalidades a los menores de 18 años de edad, a la mujer embarazada y a los causantes discapacitados mentales de cualquier edad, que no perciban una PASIS.

El cuadro 3 da cuenta de esta distribución. Cabe señalar que las personas calificadas en FONASA A carecen de recursos; en FONASA B, perciben menos de un salario mínimo vital; en FONASA C, desde un salario mínimo vital a uno y medio y, en FONASA D, más de un salario y medio mínimo. A fines de 2005, ese salario mínimo ascendía, aproximadamente, a US\$ 210.

En cambio, el número de afiliados a las ISAPRE se redujo en 31\% entre 1997 y 2004 (24). Esto obedece a la selección o "descreme" de mercado que se manifiesta en la constante alza de los valores de los planes ofrecidos a quienes tienen enfermedades crónicas o preexistentes; son de mayor edad o, en general, corren más riesgos. Dicha situación es predecible, dado el carácter lucrativo de las ISAPRE.

El cuadro 4 presenta ejemplos de las diferencias que existen entre el seguro público y el privado, en cuanto a la proporción de personas económicamente necesitadas o en situaciones de salud muy costosas.

En conclusión, en Chile las mayores responsabilidades para universalizar la cobertura de salud y, por tanto, la protección social horizontal, han sido asumidas por el seguro público. Las aseguradoras privadas hacen primar el criterio de rentabilidad antes que el de cobertura.
CUADRO 3. Afiliados a FONASA según tramo de ingresos y categoría de afiliación (diciembre 2005)

\begin{tabular}{lrr}
\hline & $n$ & $\%$ \\
\hline Tramo de ingresos & & \\
FONASA A & 3818674 & 34,3 \\
FONASA B & 3448513 & 31,0 \\
FONASA C & 1876586 & 16,9 \\
FONASA D & 1976227 & 17,8 \\
$\quad$ Total & 11120000 & 100,0 \\
Categoría de afiliación & & \\
Total de cotizantes & 4090423 & 36,8 \\
$\quad$ Dependiente & 2665565 & 24,0 \\
$\quad$ Independiente & 129920 & 1,2 \\
$\quad$ Pensionado & 1294938 & 11,6 \\
Cargas familiares & 3210903 & 28,9 \\
Total de no cotizantes & 3818674 & 34,3 \\
$\quad$ Carentes de recursos & 2396348 & 21,5 \\
$\quad$ Subsidios familiares & 964585 & 8,7 \\
Pensiones asistenciales & 457741 & 4,1 \\
\hline
\end{tabular}

Fuente: Subdepartamento de Estudios, FONASA.

CUADRO 4. Algunos indicadores comparativos de afiliados a FONASA e ISAPRE. Chile, 2004

\begin{tabular}{lll}
\hline \multicolumn{1}{c}{ Indicador } & FONASA & ISAPRE \\
\hline $\begin{array}{lll}\text { Valor promedio de las } \\
\text { cotizaciones anuales (en } \$ C h)\end{array}$ & & \\
$\quad$ por cotizante & $\$ 134000^{\mathrm{a}}$ & $\$ 831228^{\mathrm{b}}$ \\
$\begin{array}{l}\text { Tratamiento de diabetes mellitus } \\
\quad \text { tipo } 2^{\mathrm{c}}\end{array}$ & $95,5 \%$ & $4,5 \%$ \\
Tratamiento de sida $^{\mathrm{c}}$ & $94,7 \%$ & $5,3 \%$ \\
Persona mayor de 65 años & 909915 & 80488 \\
\hline
\end{tabular}

Fuente: Ministerio de Salud (25).

${ }^{a}$ Calculado en base al $7 \%$ del valor promedio de los ingresos de diciembre de 2004 contabilizados por FONASA y expandidos anualmente.

${ }^{b}$ Fuente: Superintendencia de Salud (36).

' Según estadísticas del plan Acceso Universal de Garantías Explíitas (AUGE), 2005

\section{COBERTURA VERTICAL: PRESTACIONES DE ACCESO UNIVERSAL}

Las estadísticas básicas de salud de Chile muestran logros significativos en eficiencia que provienen de cambios institucionales que se iniciaron a mediados del siglo pasado y que se han intensificado en los últimos decenios. Por ejemplo, la baja tasa de mortalidad infantil de Chile homogéneamente distribuida entre estratos socioeconómicos diferentes en el año 2000 le mereció ocupar el primer lugar en la clasificación mundial de la OMS sobre igualdad de la supervivencia infantil (26) pese a las notables mejorías en los indicadores de salud generalmente aceptados como relevantes, prácticamente todos los problemas de salud más acuciantes de la actualidad en Chile tienen un comportamiento desigual, afectando preferentemente a las personas más pobres, las que tienen menos años 
de escolaridad y las de mayor edad. La Encuesta Nacional de Salud de 2003 (27) demuestra que los problemas sanitarios están desigualmente repartidos en el país. Esto parece reflejar inequidades en la administración de la asistencia sanitaria y protección social variable según los estratos.

A continuación se examinarán especialmente las prestaciones que actualmente tienen cobertura universal, es decir, aquéllas que no deberían significar resultados diferentes en los estados de salud. ${ }^{3}$

\section{Atención de urgencia}

Según las disposiciones legales vigentes, tienen acceso a los servicios de urgencia todas las personas que los necesiten, sin importar si son beneficiarias del sistema público de salud o del lugar donde se encuentren. ${ }^{4}$ El Decreto 896, de 1999, del Ministerio de Salud precisa que urgencia o emergencia "es toda condición de salud o cuadro clínico que implique riesgo vital y/o secuela funcional grave para una persona de no mediar atención médica inmediata e impostergable. [ . . . ] La condición de salud o cuadro clínico de emergencia o urgencia debe ser determinado por el diagnóstico efectuado por un médico cirujano en la unidad de urgencia pública o privada en que la persona sea atendida, lo que deberá ser certificado por éste". En realidad, esta definición restringida a una opinión profesional surge como medida precautoria ante la detección de una proporción significativa de demanda en los servicios de urgencia que no tiene fundamento clínico sino que, más bien, obedece a insuficiencia de oferta en la red de atención primaria. En 2004 se registraron 13560000 atenciones médicas en servicios de urgencia.

Complementariamente, a fines de la década de los ochenta, se crearon los servicios de atención primaria de urgencia (SAPU), que tuvieron su apogeo en la década siguiente (13 en 1990 y 152 en 2004). Éstos son establecimientos de salud que atienden durante los horarios en que los consultorios habituales no lo hacen. Forman parte de la red de urgencia que presta atención inicial en casos de suma gravedad. De hecho, esta modalidad cubrió 29\% de las atenciones médicas en 2004.

Los objetivos que persigue el SAPU son

- aumentar la capacidad resolutiva del nivel primario de atención;

\footnotetext{
3 Salvo las que señala Sen (38): “Los factores que pueden contribuir a los logros y fracasos en el campo de la salud van mucho más allá de la atención sanitaria e incluyen muchas influencias muy distintas, desde las predisposiciones genéticas, los ingresos individuales, y los estilos de vida hasta el entorno epidemiológico y las condiciones de trabajo."

4 Página internética (web) del Ministerio de Salud visitada el 20 de enero de 2006.
}

- aumentar la cobertura de atención del consultorio;

- mejorar la accesibilidad a la atención médica de la comunidad;

- disminuir las consultas de urgencia en el nivel terciario de atención;

- disminuir los tiempos de espera por consultas de urgencia, y

- disminuir los costos por traslados debido a consultas de urgencia.

\section{Atención primaria de salud}

Los chilenos tienen una cobertura asegurada en la atención primaria de salud (APS) constituida por cinco programas principales: Salud del Niño y del Adolescente; Salud del Ambiente; Salud Maternoperinatal; Salud del Adulto y Odontológica y Programa de Laboratorios Básicos. En este nivel se efectúan alrededor de 17 millones de consultas médicas anuales (24). No obstante, $20 \%$ de las personas inscritas en la APS municipalizada acuden a prestadores privados y no usan sus derechos a prestaciones homologables gratuitas en el sector público (28). Si se considera, además, que el promedio de los copagos que asume el paciente asciende a $62 \%$ del costo de este tipo de prestaciones, su uso sólo puede explicarse por ausencia de oportunidad o de calidad en la oferta pública, al menos para ese $20 \%$ que opta por los servicios privados. De hecho, esta elección señala la existencia de dificultades para satisfacer la demanda en las condiciones actuales del sistema público.

\section{Plan de Acceso Universal de Garantías Explícitas}

Hay una abundante literatura sobre el plan de Acceso Universal de Garantías Explícitas (AUGE) y la ley que lo sustenta, conocida como Garantías Explícitas en Salud (GES), que puede ser consultada como referencia básica para esta sección (29-32). No obstante, aquí se quiere hacer referencia exclusiva a los cambios que, como consecuencia de esa ley, fortalecen la universalidad de acceso a prestaciones de salud.

El sistema de salud público nacional otorga a todos los chilenos universalidad formal de acceso a la atención de salud. No hay exclusión para las personas inscritas en el seguro público por ninguna condición, sea étnica, de ingresos, de sexo u otra. Sin embargo, en la realidad, la exclusión se produce por vía de las listas de espera, por la menor calidad, aparente o real, de las prestaciones y por los requisitos financieros necesarios, especialmente para solventar intervenciones complejas quirúrgicas o catastrófi- 
cas. En otras palabras, la oportunidad, la calidad y el financiamiento son las variables de ajuste que restringen el acceso real a las prestaciones.

En el plan AUGE (anexo 1) se han establecido las garantías explícitas de atención a 56 problemas de salud, aplicables entre 2005 y 2007. Se intenta así eliminar los ajustes espurios, ya que el plan exige que los prestadores públicos y privados cumplan con plazos perentorios, definidos de acuerdo con criterios sanitarios, para otorgar al paciente las prestaciones mínimas (el indicador de calidad actualmente vigente) ${ }^{5}$ que permiten obtener resultados clínicos. El criterio principal que permite diferenciar esta situación de la anterior es que la reforma de salud precisa los tiempos de espera, que deben cumplirse en plazos perfectamente conocidos por la población. ${ }^{6}$ Por su parte, la especificación de las prestaciones mínimas que requieren los problemas de salud incluidos en el AUGE en las cuatro fases fundamentales, es decir, sospecha, diagnóstico, tratamiento y seguimiento, es también de acceso público (anexo al Decreto 170). Todo lo anterior fue ratificado por el Ministerio de Hacienda que, conjuntamente con el Ministerio de Salud, emitió el Decreto 228 de 2005. Esto tiene la importancia de asegurar los recursos para las garantías comprometidas.

A continuación, se presentan ejemplos de los plazos en que deben realizarse las prestaciones para un problema crítico, como es el infarto agudo del miocardio (IAM). De acuerdo con la ley, tienen derecho a diagnóstico los beneficiarios que presenten dolor torácico no traumático o síntomas de IAM. Tendrá derecho a tratamiento médico y seguimiento quien tenga diagnóstico confirmado y tendrán derecho a seguimiento quienes hayan sido sometidos a revascularización coronaria o angioplastia coronaria percutánea.

En cuanto a la oportunidad, el decreto ministerial estipula que los pacientes con sospecha de diagnóstico de infarto tienen que recibir atención médica de urgencia dentro de un plazo de 30 minutos. Luego, en los tratamientos con confirmación diagnóstica, las medidas generales deben ser aplicadas dentro de las 24 horas siguientes. Otra exigencia perentoria en el caso específico de pacientes

\footnotetext{
La ley 19.966 define como "garantía explícita de calidad" que los prestadores de la atención de salud sean registrados o acreditados, de acuerdo con la ley 19.937, en la forma y condiciones que determine el decreto a que se refiere el artículo 11. En consecuencia, no se alude directamente a las prestaciones sino a las características o requisitos de los prestadores. Empero, en los hechos, el plan AUGE enumera prestaciones y medicamentos, y registra parámetros mínimos con los que deben cumplir las coberturas. Según la opinión de expertos, el objeto de no transformar esos procedimientos, insumos y valores en criterios uniformes de calidad es evitar descartar tratamientos e insumos "alternativos, pero no clínicamente inferiores".

Éstos aparecen estipulados en documentos de acceso público como el Decreto 170 del Ministerio de Salud con el Listado de Prestaciones Específico de Garantías Explícitas en Salud, publicado en el Diario Oficial del 28 de enero de 2005.
}

con elevación del segmento ST e indicación de trombólisis es que deben ser tratados dentro de 30 minutos a partir de la confirmación diagnóstica por electrocardiograma. Además, es preciso asegurar su hospitalización y tratamiento médico de urgencia dentro de seis horas. Por último, en el marco del seguimiento se proyecta un primer control del paciente para prevención secundaria, dentro de 30 días después del alta de la hospitalización. Puede entonces sometérsele a algún tratamiento médico indicado para IAM, a revascularización o angioplastia coronaria percutánea.

En conclusión, las ventajas de que los sistemas de salud incorporen la cobertura universal garantizada de problemas o estados patológicos - según criterios que apunten a mejorar la salud de las personas o su bienestar general-, justifican las dificultades que conlleva establecer metodologías consensuadas para establecer las listas mencionadas. En consecuencia, la inclusión gradual de los 56 problemas en el plan AUGE es un paso importante hacia la universalidad de la atención de la salud y algo que podrá ampliarse y profundizarse en el futuro.

\section{PROTECCIÓN SOCIAL Y PROTECCIÓN FINANCIERA}

\section{Fuentes de financiamiento de la salud}

Se podría esperar que a mayor gasto se obtuvieran mejores estados de salud de la población. En algunos textos se estima que la esperanza de vida podría ser el indicador global de eficiencia en el uso de los fondos destinados a la atención de salud. En ese caso, según la Organización para la Cooperación y el Desarrollo Económico (OCDE, 33), los japoneses, con una esperanza de vida de 82 años obtienen la mayor efectividad del mundo de sus gastos en salud. En cambio, los Estados Unidos de América, pese a gastar cerca de $15 \%$ del PIB en salud, alcanzan apenas el lugar 22 en orden de importancia, con una esperanza de vida de 77 años. Corea del Sur, que gasta sólo 5,6\% del PIB, alcanza también los 77 años en esperanza de vida. En Chile, por su parte, con un gasto de 5,9\% del PIB (US\$ 4500 millones anuales, aproximadamente, y US\$ 320 per cápita), el indicador de esperanza de vida se ubica también en 77 años. En definitiva, pareciera que el nivel y cuantía de los gastos en salud no está correlacionado con los resultados sanitarios. En realidad, lo anterior sugiere una discusión que no puede profundizarse en esta oportunidad; a saber, que si los aumentos de gastos están muy focalizados en grupos minoritarios, los impactos sobre las estadísticas nacionales pueden ser irrelevantes. 
CUADRO 5. Chile: estadísticas económicas de salud básicas

\begin{tabular}{|c|c|c|}
\hline & \multicolumn{2}{|c|}{ Porcentaje } \\
\hline & 2003 & 2004 \\
\hline Aporte público directo & 28,8 & 32,1 \\
\hline Aporte fiscal & 27,2 & 30,6 \\
\hline Aporte municipal & 1,6 & 1,6 \\
\hline Aporte público indirecto & 38,3 & 37,5 \\
\hline Cotizaciones de la seguridad social: & & \\
\hline Al seguro público & 17,4 & 16,7 \\
\hline Al seguro privado (obligatorias) & 20,9 & 20,8 \\
\hline Gastos de bolsillo & 33,0 & 30,4 \\
\hline Cotizaciones voluntarias & 6,7 & 6,7 \\
\hline Copagos por prestaciones privadas & 9,5 & 9,5 \\
\hline Otros pagos (no afiliados) & 3,8 & 2,1 \\
\hline Medicamentos & 13,0 & 12,1 \\
\hline Gasto total en salud & 100,0 & 100,0 \\
\hline Porcentaje del PIB gastado en salud & 5,6 & 5,9 \\
\hline Gasto total en salud (en millones de US\$ corrientes) ${ }^{a}$ & 4,044 & 5,121 \\
\hline Gasto total en salud (en millones de US\$ corregidos de 2003) & 4,044 & 4,508 \\
\hline Tasa de crecimiento del gasto en salud en pesos corrientes & & 13,1 \\
\hline Tasa de crecimiento del gasto en salud en dólares al valor de 2003 & & 11,5 \\
\hline Tasa de crecimiento del PIB, 2004 & & 6,1 \\
\hline Inflación, 2004 & & 2,4 \\
\hline Tasa de crecimiento de la proporción salud/PIB & & 5,5 \\
\hline
\end{tabular}

a Tipo de cambio medio anual según el Banco Central de Chile: en 2003, US\$1=\$Ch691,4; en 2004, US\$1= $\$ C h 609,53$.

Para el financiamiento de la salud en Chile, en 2004 existían tres fuentes principales: aportes fiscales $(32,1 \%)$, cotizaciones obligatorias a la seguridad social $(37,5 \%)$ y gastos de bolsillo $(30,4 \%)$, incluidos los seguros privados no obligatorios. El cuadro 5 da cuenta de los cambios efectuados en 2004 para financiar la reforma de salud. Por otra parte, el aporte fiscal ha aumentado en alrededor de $30 \%$ - en términos reales- entre 2000 y 2005, lo que indica que las reformas de salud difícilmente podrán reducir las brechas de equidad sin utilizar recursos del presupuesto público.

En cuanto a la distribución de recursos entre el seguro público y los seguros privados, en 2004 FONASA dispuso de $\$ C h 121960$ anuales por beneficiario (US\$ 200 al cambio promedio anual), de los cuales $48 \%$ corresponden a aportes públicos directos. Otro $42 \%$ corresponde a cotizaciones, $8 \%$ a copagos y $2 \%$ a otros ingresos (35). En el mismo año, las ISAPRE dispusieron del equivalente a US $\$ 495$ por beneficiario (36). ${ }^{7}$ De éstos, $71 \%$ correspondieron a cotizaciones obligatorias, $26 \%$ a cotizaciones voluntarias adicionales y $3 \%$ a los aportes de empleadores. ${ }^{8}$

\footnotetext{
7 Estas cifras son diferentes de las del cuadro 3 porque allí se comparan los cotizantes y aquí los beneficiarios. Esto significa que en este último caso se incluyen los aportes fiscales al FONASA y las cargas por persona.

8 Se trata esencialmente de aportes compensatorios que hacen los empleadores en los convenios grupales.
}

Si bien es difícil establecer un monto o proporción razonable para los gastos de bolsillo, la OMS indica que son de todos modos imprescindibles: "Aunque el pago anticipado es una piedra angular de un financiamiento equitativo de un sistema de salud, en los países o entornos de bajos ingresos tal vez se requiera alguna contribución directa en el momento de la utilización para aumentar los ingresos cuando no hay suficiente capacidad de pago anticipado" (26). Como sea, será necesario estudiar si en Chile éstos cumplen con las condiciones básicas de protección social que señala Wagstaff (37): a) que la distribución de los costos de la atención sanitaria no aumente la desigualdad de los ingresos; b) que las familias no gasten en tales atenciones más allá de un porcentaje determinado de sus ingresos (40\% es el guarismo de uso común en la OPS); c) que los costos de la atención sanitaria no lleven a las familias a la pobreza o a aumentarla; y conviene agregar un punto d) que no se exacerben las diferencias en los estados de salud de los grupos sociales como consecuencia de una insuficiencia de ingresos para financiar la atención sanitaria con gastos de bolsillo.

Como es sabido, los medicamentos y los copagos por prestaciones son los elementos centrales de gastos entre los asegurados. La variación en el precio de los medicamentos y su importancia cre- 
CUADRO 6. Porcentaje empleado en medicamentos del total de gastos en salud de los hogares, según decil, 1997

\begin{tabular}{cccccccccccc}
\hline $\begin{array}{c}\text { Gastos } \\
\text { en medicamentos }\end{array}$ & \multicolumn{11}{c}{ Gastos por decil } \\
\hline Total & I & II & III & IV & V & VI & VII & VIII & IX & X \\
27,2 & 57,6 & 43,0 & 41,4 & 44,1 & 39,6 & 35,9 & 32,5 & 31,6 & 20,4 & 19,5 \\
\hline
\end{tabular}

Fuente: Instituto Nacional de Estadística (37). Elaboración del autor.

ciente en la estructura de los costos de la atención de salud son temas sobresalientes de los trabajos que examinan los aspectos económicos de la salud. En el conjunto de países de la OCDE, los gastos en fármacos han aumentado en promedio $32 \%$, en términos reales, entre 1998 y 2003. En este último año, el total ascendió, aproximadamente, a US\$ 450000 millones (33).

En Chile, el expendio en medicamentos constituye $17 \%$ de los gastos totales en salud. El presupuesto público cubre sólo una cuarta parte de ese costo, mientras que los seguros privados, en general, no cubren este rubro y la diferencia debe ser pagada directamente por los pacientes. También es cierto que, al igual que en otros países, los precios de los bienes y servicios de la salud aumentan más rápidamente que los precios generales de los bienes y servicios que requieren los hogares.

El cuadro 6 fue elaborado a partir de la última encuesta nacional de presupuestos familiares realizada por el Instituto Nacional de Estadísticas (INE) en 1997 y muestra los gastos directos de los hogares en medicamentos. Puesto que la mayor parte de éstos no son cubiertos por la seguridad social (pública o privada), en este gasto se observa regresividad, es decir, que los grupos de menores ingresos dedican una proporción mayor a medicamentos (57,6\% en el primer decil) que los grupos de mayores ingresos (19,5\% en el décimo decil). Podría concluirse, en cambio, que los pobres tienen menos gastos en las demás prestaciones, lo cual es cierto porque generalmente se atienden en el sector público en el cual no tienen copagos. Sin embargo, cabe recordar que las listas de espera - que retrasan el acceso oportuno- son una variable de ajuste en el sector público, que perjudica a los más pobres ya que éstos, por falta de recursos, no tienen la opción de hacerse atender privadamente.

\section{La cobertura financiera del seguro médico público (bonificaciones y copagos)}

Criterios generales. En Chile, el seguro médico público otorga cobertura financiera según el tramo de ingreso del cotizante o beneficiario. Las personas y sus familiares a cargo en el tramo A, correspondiente a carentes de recursos, tienen acceso gratuito. Las del tramo B (con ingresos inferiores al mínimo vital) tienen también copago cero; las del tramo $\mathrm{C}$ (al menos ingresos iguales a un mínimo vital pero inferior a 1,5 veces ese valor) tienen copago equivalente a $10 \%$ del valor de las prestaciones; y las del tramo D (ingresos superiores al anterior) copagan $20 \%$. Cabe señalar que existe una garantía financiera adicional tal que los cotizantes de los grupos $\mathrm{D}$ y C con más de tres familiares a cargo son trasladados a los grupos C y B, respectivamente.

No obstante, hay varias excepciones a estas reglas de copago. Un ejemplo es el grupo 27 del arancel de la Modalidad de Atención Institucional (MAI), compuesto especialmente por atenciones odontológicas en que los copagos son $30 \%$ para el grupo B; $50 \%$ para el grupo C; y $80 \%$ para el grupo D. Tampoco se cumple la regla general en gran parte de las atenciones de consulta de especialidades en la Modalidad de Libre Elección (con prestadores privados) en que los copagos superan dichos valores.

Cobertura financiera del plan AUGE. Por su parte, el plan AUGE establece protección financiera explícita universal para todos los asegurados, tanto por empresas privadas como por el seguro público. La contribución del afiliado por prestación o grupo de prestaciones no deberá exceder un máximo de $20 \%$ del valor determinado en un arancel de referencia del régimen, con las reducciones que contempla FONASA para los grupos A, B y C.

Uno de los factores más importantes de protección social quedó plasmado en el artículo 5 de la Ley 19.966 que define la cobertura financiera adicional como el financiamiento de $100 \%$ de los copagos originados sólo por enfermedades o condiciones de salud contenidas en las Garantías Explícitas en Salud que superen el deducible. (Éste es la suma de los copagos que habrán de ser acumulados por cada evento para tener derecho a la cobertura financiera adicional.) Específicamente, el deducible equivale a 29 cotizaciones mensuales legales o pactadas. A modo de referencia, para personas con ingresos estables y permanentes durante un año en el grupo D, el pago máximo no debe superar 25\% del ingreso. 
CUADRO 7. Chile: beneficiarios del seguro catastrófico. Datos de FONASA, 1999-2004

\begin{tabular}{|c|c|c|c|c|c|c|}
\hline Área de atención & 1999 & 2000 & 2001 & 2002 & 2003 & 2004 \\
\hline Cardiocirugía $^{a}$ & 2730 & 2933 & 3377 & 4267 & 7745 & 9710 \\
\hline Neurocirugía & 1787 & 1952 & 2257 & 3355 & 6052 & 10210 \\
\hline Trasplante renal ${ }^{b}$ & 233 & 248 & 218 & 225 & 221 & 236 \\
\hline Trasplante hepático & 24 & 27 & 27 & 26 & 26 & 36 \\
\hline Diálisis peritoneal ${ }^{b}$ & 45 & 55 & 61 & 64 & 72 & 91 \\
\hline Radioterapia $^{a}$ & 4492 & 4499 & 4758 & 5096 & 4568 & 7848 \\
\hline Programa nacional de drogas antineoplásicas ${ }^{a}$ & 104 & 82 & 103 & 956 & 944 & 1172 \\
\hline Fármaco inmunosupresorb & 1281 & 1418 & 1639 & 1793 & 2111 & 2145 \\
\hline Escoliosis $^{b}$ & 251 & 205 & 212 & 263 & 277 & 425 \\
\hline Fisuras palatinas $^{b}$ & 428 & 595 & 740 & 941 & 1008 & 1427 \\
\hline Quemadura grave & 196 & 271 & 218 & 218 & 212 & 221 \\
\hline Politraumatismos & 88 & 87 & 86 & 82 & 52 & 68 \\
\hline Cuidados paliativos $^{b}$ & 4065 & 5662 & 5772 & 6583 & 1142 & 12898 \\
\hline Hemodiálisis ${ }^{b}$ & 63 & 802 & 823 & 1229 & 1779 & 2549 \\
\hline Trasplante de médulab ${ }^{b}$ & 4 & 11 & 15 & 18 & 18 & 20 \\
\hline Quimioterapia, cáncer de mama ${ }^{b}$ & 193 & 518 & 756 & 1169 & 1546 & 1830 \\
\hline Sida ${ }^{b}$ & 0 & 0 & 1657 & 3308 & 4038 & 5700 \\
\hline Quimioterapia, cáncer cervicouterinob & 0 & 0 & 0 & 626 & 469 & 602 \\
\hline Vitrectomía & 0 & 0 & 0 & 740 & 818 & 858 \\
\hline Fibrosis quística & 0 & 0 & 0 & 225 & 280 & 292 \\
\hline Cirugía por cáncer preinvasor e invasor del cuello uterinob & 0 & 0 & 0 & 0 & 7902 & 8104 \\
\hline Total & 15984 & 19365 & 22719 & 31184 & 51566 & 66442 \\
\hline
\end{tabular}

Fuente: FONASA, Departamento de Comercialización.

a En 2005, cubierto en parte por AUGE y otra parte por el Seguro Catastrófico.

${ }^{\mathrm{b}}$ Cubierto por el plan AUGE en 2005.

Programas especiales del seguro público y su cobertura financiera. Con anterioridad a la implantación del plan AUGE en 2005, existían programas especiales del FONASA con cobertura financiera adicional, que se han integrado gradual o parcialmente al AUGE:

El seguro catastrófico. Este seguro funciona desde 1999 y cubre $100 \%$ del financiamiento de la atención de enfermedades complejas y de alto costo tales como el cáncer, cardiopatías complejas, derrames y tumores cerebrales. En el transcurso de 1999 a 2004, como se indica en el cuadro 7 , se acogieron a este beneficio alrededor de 200000 personas (cerca de $2 \%$ de los asegurados por FONASA).

El Programa Adulto Mayor. Orientado a mejorar la funcionalidad de los adultos mayores, aumenta la cobertura financiera de aquellas dolencias que afectan con mayor frecuencia a este segmento. Incluye financiamiento para ortesis y prótesis que mejoran su calidad de vida. El Programa ha sido implementado en la MAI para mayores de 65 años, pero en marzo de 2006 se amplió a los mayores de 60 años y en la Modalidad de Libre Elección ${ }^{9}$ para mayores

\footnotetext{
9 Los afiliados al seguro público tienen dos opciones para recibir sus prestaciones: en la modalidad de atención institucional (MAI), es decir, la red total de prestadores públicos (consultorios y hospitales); $y$, en la modalidad de libre elección (MLE) conformada por prestadores privados. A esta última tiene acceso solo una parte de los beneficiarios y la cobertura financiera (bonificación) sólo representa una fracción del costo fijada por aranceles en FONASA.
}

de 55 años. En 2005, 62973 personas se beneficiaron de este Programa.

Entre los beneficios especiales que pueden obtener los afiliados de este Programa para mayores, aún atendiéndose con prestadores privados, se destacan:

- Bonificación de lentes ópticos y audífonos $(100 \%)$;

- Atención integral de enfermería a domicilio (50\% de cobertura), y

- Atención integral de enfermería en centros del adulto mayor ( $50 \%$ de cobertura).

El Programa Oportunidad en la Atención. Desde 1996, este programa procura complementar la capacidad de oferta del sector público recurriendo al sector privado, reduciendo las listas de espera y fijando bonificaciones adicionales para prestaciones, generalmente quirúrgicas. En 2005 hubo 32871 beneficiarios.

Las atenciones de urgencia. Desde 1999 esas atenciones fueron beneficiadas con la disposición legal que "prohíbe a los prestadores exigir a los beneficiarios de esta ley, dinero, cheques u otros instrumentos financieros para garantizar el pago o condicionar de cualquier otra forma dicha atención" (Ley 18.459). En el mismo cuerpo legal se establece que estas atenciones podrán ser bonificadas, a lo menos en $60 \%$ y hasta $90 \%$. 
Programa de Reparación y Atención Integral en Salud y Derechos Humanos (PRAIS). Los beneficiarios de este programa destinado a los detenidos y víctimas de la represión entre 1973 y 1990 y sus familias

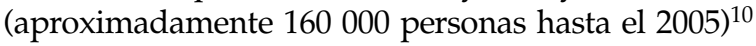
acceden automáticamente al grupo A de FONASA y, por lo tanto, también tienen un copago igual a cero.

\section{Conclusiones}

El concepto de protección social de la salud se especifica en este texto a través de tres dimensiones e indicadores principales:

- La cobertura horizontal o proporción de la población que tiene cobertura de la seguridad social en salud;

- La cobertura vertical o prestaciones a las que tienen derecho efectivo, oportuno y razonablemente garantizado tales afiliados, y

- La protección financiera o medida para que los gastos totales o complementarios en salud no exacerben la desigualdad en ingresos o estados de salud.

En Chile, como en toda América Latina, se observa una reducción de la proporción de trabajadores dependientes. A ello se suma el aumento del trabajo por cuenta propia; la contratación externa (outsourcing) como estrategia creciente de las grandes empresas y el incremento de los empleos a tiempo parcial y de los empleos temporales. En suma, la persistencia de un amplio sector informal así como la precarización de los empleos atentan contra la universalidad de la seguridad social basada en cotizaciones de trabajadores dependientes, lo que obliga a una participación y responsabilidad directa del Estado para financiar la salud de la población.

En efecto, los impactos financieros sobre la seguridad social son inmediatos. Las estadísticas de cotizaciones de FONASA indican que, como consecuencia de esta precariedad de los empleos, sólo $25 \%$ de la población trabajadora afiliada paga sus cuotas por el año completo.

Por otra parte, la ampliación de la cobertura horizontal de la seguridad social ha producido un comportamiento disímil entre el seguro público y el privado. El seguro público ha mejorado la legislación a favor de los grupos desprotegidos ampliando la cobertura de

- los trabajadores dependientes con contrato temporal por obra, faena o turnos;

\footnotetext{
10 Muchos de ellos están cubiertos de todos modos por el seguro público, por ser trabajadores o pensionados.
}

- los cesantes;

- las personas carentes de recursos (indigentes);

- los familiares a cargo de los afiliados (esta condición también es válida en el seguro privado);

- los beneficiarios de pensiones asistenciales de invalidez y ancianidad o del subsidio único familiar;

- las personas con deficiencia mental;

- la mujer embarazada hasta el sexto mes del nacimiento del hijo;

- las atenciones de urgencia, y

- las víctimas de la represión en la dictadura (PRAIS).

En cambio, el número de afiliados de las ISAPRE se redujo en 31\% entre 1997 y 2004, lo cual obedeció principalmente a la selección o "descreme" de mercado que se manifiesta por la constante alza del valor de los planes de seguros de quienes tienen enfermedades crónicas o preexistentes, son de mayor edad o, en general, están en mayor riesgo de enfermar.

Pese a los notables logros generales en cuanto a indicadores trascendentales de la salud en Chile, en la actualidad prácticamente todos los problemas de salud más acuciantes tienen un comportamiento desigual, afectando preferentemente a los más pobres, a los que tienen menos años de escolaridad y a los de mayor edad. Esto produce inequidades en la administración de la asistencia sanitaria y desigual protección social según los estratos.

En la actualidad, ciertas prestaciones tienen cobertura universal, como las de APS y las de urgencia. No obstante, $20 \%$ de las personas inscritas en la APS pública acuden a prestadores privados y no usan sus derechos a prestaciones homologables gratuitas en el sector público. Si se considera, además, que el promedio de los copagos que asume el paciente es de $62 \%$ del costo de este tipo de prestación, su uso sólo puede explicarse por ausencia de oportunidad o de calidad en la oferta pública, al menos para ese $20 \%$ que opta por modalidades privadas, lo que reduce la universalidad efectiva de estas prestaciones.

La inclusión gradual en Chile de los 56 problemas de salud listados en el plan AUGE es un paso importante hacia una profundización de la cobertura vertical, que podrá ampliarse en el futuro. El valor principal de este plan es que fija, por un lado, plazos perentorios y conocidos para asegurar la oportunidad de la atención y, por otro, guías clínicas de referencia que establecen pautas para determinar la calidad. Asimismo, asegura la protección financiera limitando la cantidad máxima de copago a $20 \%$ de los valores establecidos por una lista arancelaria. Otra garantía financiera adicional es el deducible que limita el copago, a fin de evitar que éste, en un año, supere alrededor de $25 \%$ de los ingresos. 
Cabe notar que el nivel y cuantía de los gastos en salud no está correlacionado con los resultados sanitarios. Chile, por ejemplo, con un gasto de 5,9\% del PIB (US\$ 4500 millones de dólares anuales, aproximadamente, y US\$320 per cápita), tiene un indicador de la esperanza de vida de 77 años, al igual que los Estados Unidos con un gasto en salud per cápita de US\$ 5635.

No obstante, se concluye que las reformas de salud en países con niveles significativos de pobreza y desigualdades sociales (incluido Chile) difícilmente lograrán reducir esas brechas para fomentar la equidad, sin destinar a ello recursos del presupuesto público. Los aportes fiscales directos, las cotizaciones y los gastos de bolsillo representan cada uno un tercio, aproximadamente, de los gastos de salud nacionales. Los gastos de bolsillo constituyen todavía una proporción crítica de desprotección que puede aumentar las desigualdades de ingresos o estados de salud, o bien conducir los hogares a la pobreza o intensificarla. En efecto, si la proporción del ingreso gastado en medicamentos es superior en los deciles más bajos, como ocurre en Chile, se verifica un impacto regresivo en este rubro.

Si bien el copago en el sector privado depende de cada plan individual, en el seguro público el copago oscila entre cero para carentes de recursos y $20 \%$ para quienes reciben más de 1,5 salarios mínimos. Sin embargo, hay excepciones que superan este porcentaje; por ejemplo, atenciones odontológicas y consultas de especialidades con prestadores privados, las cuales suelen justificarse por la insuficiente capacidad de oferta del sector público.

Finalmente, cabe notar que el sector público, principalmente desde la década de los años noventa, ha puesto en marcha iniciativas que mejoran la cobertura horizontal y vertical de la protección social, especialmente el plan AUGE. Pero antes de él, se desarrollaron programas de cobertura parcial con notorias ventajas financieras para atenciones de urgencia, enfermedades catastróficas para adultos mayores y para incluir a prestadores privados en los casos que la insuficiente oferta pública generaba largas listas de espera. Estas experiencias fueron de gran utilidad para universalizar estos beneficios posteriormente.

\section{SYNOPSIS}

\section{Chile: social protection in health}

This piece begins with a brief discussion of the concepts leading to the social right to health protection. Special emphasis is placed on the principle of social cohesion, which has influenced social health protection in European countries. Chile's experience in this field from the 1990s to the present is described, as exemplified in three dimensions. In the first place, social security coverage is presented as a means to achieve universal (horizontal) coverage. A discussion follows on vertical coverage, where the author identifies health problems for which insured persons have guaranteed rights of access to medical care. This section describes available emergency care, primary health care, and the special plan for Universal Access to Explicit Guarantees (Acceso Universal de Garantías Explícitas de salud, or AUGE). Thirdly, the discussion covers the funding sources supporting the Chilean health care system: Government subsidies, contributions to social security, and out-of-pocket disbursements for private care. Chile's public health system has various special programs. One of them is catastrophic insurance, which covers $100 \%$ of the care needed for complex and very costly treatments. Older persons (over 65) have coverage for $100 \%$ of the cost of eyeglasses and hearing aids, and for 50\% of the cost of home care. If life expectancy is an appropriate indicator of health system results, it is worth noting that Chile and the United States of America have both achieved a life expectancy of 77 years, even though Chile spends only $5.9 \%$ of its gross domestic product on health care, as compared to the $15 \%$ spent by the United States.

Key words: universal coverage; health care costs; health services accessibility; economics; insurance, health; Chile.

\section{REFERENCIAS}

1. Artigas C. Una mirada a la protección social desde los derechos humanos y otros contextos internacionales. Serie Políticas Sociales. Santiago: CEPAL; 2005.

2. Zúñiga F. XI tesis del estado social y democrático de derecho: el Estado regulador y la actividad económica. Colección Ideas 3/22. Santiago: Fundación Chile; 21:2002.

3. Stiglitz J. El rumbo de las reformas: hacia una nueva agenda para América Latina. Rev CEPAL. 2003;80:7-40.

4. Williamson J. The Washington consensus as policy prescription for development. Washington DC: Institute for International Economics; 2004.
5. Williamson J. Our agenda in the Washington consensus. Washington DC: Institute for International Economics; 2003.

6. Holzmann R, Jorgensen S. Social protection as social risk management: conceptual underpinnings for the social protection sector. Strategy Paper, SP Discussion Paper 9904. Washington DC: World Bank; 1999.

7. Hicks N, Wodon Q. Protección social para los pobres en América Latina. Rev CEPAL. 2001;73:95-116.

8. World Bank. Household risk management and social protection in Chile. Washington DC: World Bank; 2004.
9. Sojo A. Vulnerabilidad social, aseguramiento y diversificación de riesgos en América Latina y el Caribe. Rev CEPAL. 2003;80:121-40.

10. Titelman D, Uthoff A. El papel del aseguramiento en la protección social. Rev CEPAL. 2003;81:103-22.

11. Somavía J. Introducción. En: Bonilla García A, Gruat JV. Protección social: una inversión durante todo el ciclo de vida para propiciar la justicia social, reducir la pobreza y fomentar el desarrollo sostenible. Ginebra: OIT; 2003.

12. Conseil de l'Europe. Stratégie de cohésion sociale du Conseil de l'Europe. Bruxelles: Conseil de l'Europe; 2004. 
13. Conseil de l'Europe. Guide méthodologique pour l'élaboration concertée des indicateurs de la cohésion sociale. Bruxelles: Conseil de l'Europe; 2005.

14. United Kingdom. National action plan on social inclusion 2003-2005: implementation report. July 2005. Disponible en http://europa.eu.int/comm/ employment_social/social_inclusion/ docs/2005/uk_en.pdf

15. Wagstaff A, Van Doorslaer E. Equity in health care finance and delivery. In: Culyer AJ, Newhouse. JP, eds. Handbook of health economics, Vol I. Elsevier Science BV; 2000.

16. Tokman V. Hacia una visión integrada para enfrentar la inestabilidad y el riesgo. Rev CEPAL. 2003;81: 81-101.

17. Banco Central de Chile (BCCh). Indicadores económicos y sociales de Chile 1960-2000. Santiago: BCCh; 2001.

18. Chile, Instituto Nacional de Estadísticas. Encuesta nacional de empleo septiembre-noviembre 2005. Disponible en http://www.ine.cl/. Acceso el 25 de enero de 2006.

19. Uthoff A. Mercados de trabajo y sistemas de pensiones. Rev CEPAL. 2002: (78):39-53.

20. Marcel M. Opciones de financiamiento público para la agenda de desarrollo. Disponible en http://www.dipres.cl/. Elaborada en noviembre 2004.

21. Chile, Ministerio de Planificación. Pobreza y distribución del ingreso en las regiones, Vol 2. Serie Casen 2003. Santiago: Mideplan-División Social; 2004.

22. Rosenberg H, Andersson B. Repensar la protección social en salud en América Latina y el Caribe. Rev Panam Salud Pública 2000;8(1/2):118-25.

23. Superintendencia de Administradoras de Fondos de Pensiones, Seguro de Cesantía. Estadísticas de afiliados, cotizantes y beneficiarios. Actualizada a noviembre de 2005. Disponible en http://www.safp.cl/. Visitada el 13 de febrero de 2006.

24. Fondo Nacional de Salud de Chile. Boletín Estadístico. Santiago: FONASA; 1998 y 2004

25. Chile, Ministerio de Salud. Cuenta Pública 2005. Presentación del Dr. Pedro García, Ministro de Salud, 10 enero 2006. Disponible en http://www.minsal.cl/ Visitado el 2 de febrero de 2006.

26. Organización Mundial de la Salud (OMS) Informe sobre la salud en el mundo 2000. Mejorar el desempeño de los sistemas de salud. Ginebra: OMS; 2000.

27. Chile, Ministerio de Salud, Departamento de Epidemiología. Resumen Ejecutivo. En: Encuesta nacional de salud, 2003. El Vigía 20, Boletín de Vigilancia en Salud Pública. Santiago: MinSal; s/f.

28. Sánchez JM. Caracterización beneficiarios de atención primaria de salud municipalizada. Documento Final. Departamento de Gestión Estratégica: FONASA; 2004. (Documento fotocopiado.)
29. Chile, Ministerio de Salud, Comisión de Reforma. Objetivos sanitarios para la década 2000-2010. Santiago: MinSal; 2002.

30. Chile, Ministerio de Salud. Sistema de acceso universal con garantías explícitas: construcción de la propuesta técnica del año base. Santiago: MinSal; 2002.

31. Sandoval H. Mejor salud para los chilenos. Cuad Med Soc. 2004;43(1); 5-16.

32. Urriola R. Financiamiento y equidad en salud: el seguro público chileno. Rev CEPAL. 2005; 87:61-78.

33. Organización para la Cooperación y el Desarrollo Económico (OCDE). OCDE in figures: statistics on the member countries. OCDE; 2005.

34. Urriola R. Cuentas de salud de Chile (resultados preliminares). Documento de trabajo. Santiago: FONASA; 2004.

35. Fondo Nacional de Salud de Chile. Cuenta pública 2004. Santiago: FONASA; 2005.

36. Superintendencia de Administradoras de Fondos de Pensiones. Resultados financieros comparados del sistema ISAPRE a diciembre 2004. Disponible en http://www.sisp.cl/. Visitada el 13 de febrero de 2006

37. Wagstaff A. Pobreza y desigualdades en el sector salud. Rev Panam Salud Publica. 2002; 11 (5/6): 316-26.

38. Instituto Nacional de Estadísticas (INE). Base de datos de la encuesta de hogares 1997. Santiago: INE

39. Sen A. ¿Por qué la equidad en salud? Rev Panam Salud Publica. 2002; 11(5/6): 302-9.

\section{ANEXO 1. Problemas sanitarios cubiertos por el plan AUGE}

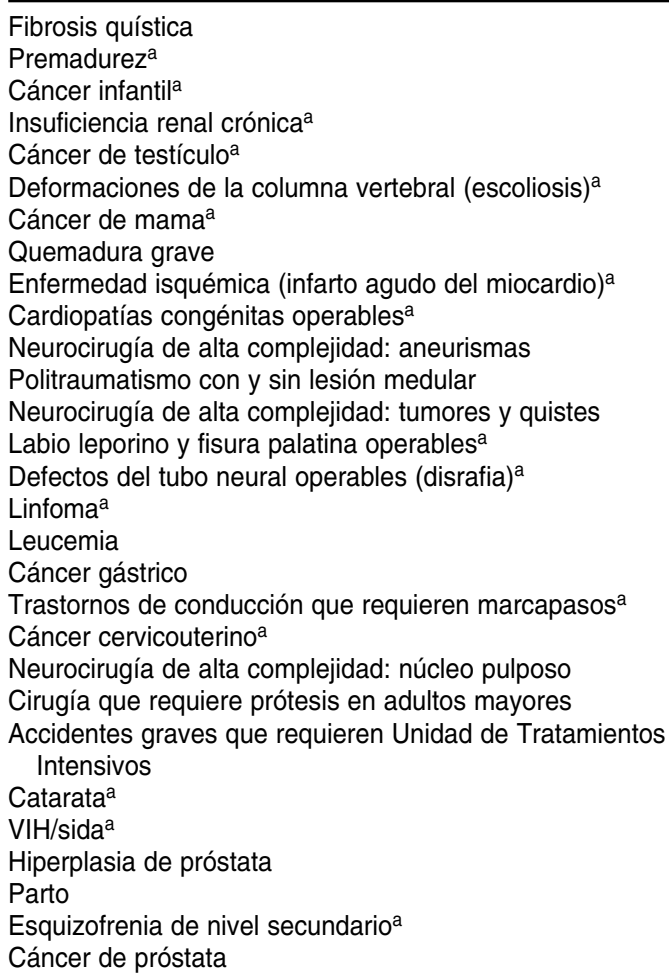

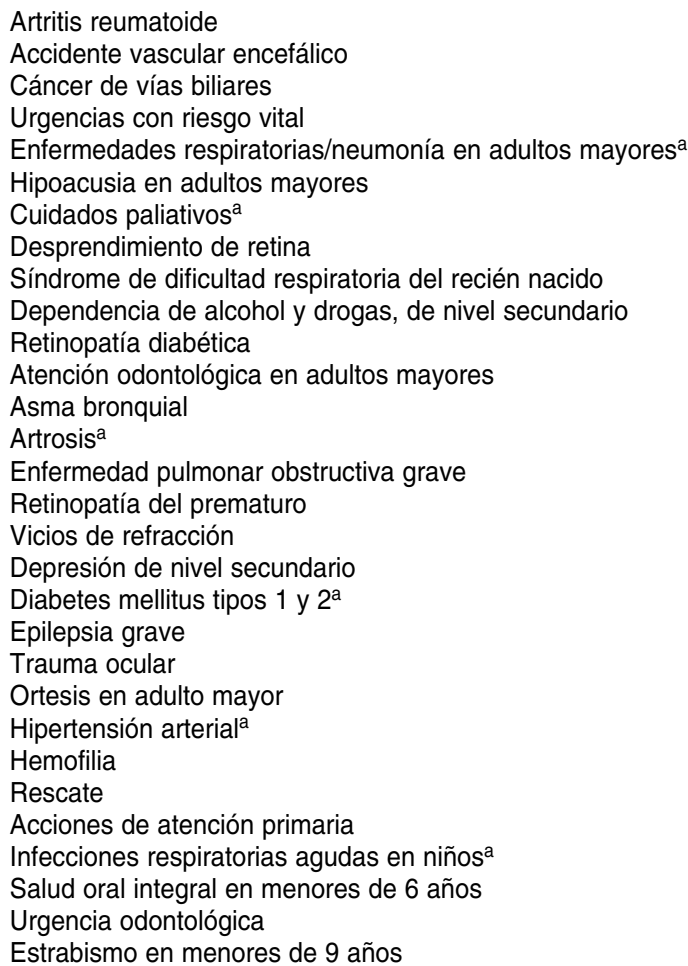

Fuente: (29).

${ }^{a}$ En vigencia desde el $1^{\circ}$ de julio de 2005. De estos 59 problemas iniciales se deberían escoger 56 hasta 2007, aunque el nuevo gobierno ofreció aumentarlos a 80 antes de 2010. 